\title{
Summary of New Insight into Electron Transport in Metals
}

\author{
Vilius Palenskis ${ }^{1}$ and Evaras Žitkevičius ${ }^{2, *(D)}$ \\ 1 Faculty of Physics, Vilnius University, Saulètekio Av. 9, 10222 Vilnius, Lithuania; vilius.palenskis@ff.vu.lt \\ 2 Department of Electronic Systems, Vilnius Gediminas Technical University, Naugarduko Str. 41, \\ 03227 Vilnius, Lithuania \\ * Correspondence: evaras.zitkevicius@vilniustech.lt
}

Citation: Palenskis, V.; Žitkevičius, E. Summary of New Insight into Electron Transport in Metals. Crystals 2021, 11, 622. https://doi.org/ $10.3390 /$ cryst11060622

Academic Editors: Pavel Lukáč and Andrei Vladimirovich Shevelkov

Received: 9 April 2021

Accepted: 28 May 2021

Published: 31 May 2021

Publisher's Note: MDPI stays neutral with regard to jurisdictional claims in published maps and institutional affiliations.

Copyright: (c) 2021 by the authors. Licensee MDPI, Basel, Switzerland. This article is an open access article distributed under the terms and conditions of the Creative Commons Attribution (CC BY) license (https:// creativecommons.org/licenses/by/ $4.0 /)$.

\begin{abstract}
This paper gives a summary of a new insight into basic electron transport characteristics in crystalline elemental metals. The general expressions based on the Fermi-Dirac distribution of the effective density of the randomly moving electrons, their diffusion coefficient, drift mobility, and other characteristics, including the Einstein relation between diffusion coefficient and drift mobility, are presented. It is shown that the creation of the randomly moving electrons due to lattice atom vibrations produces the same number of electronic defects, which cause scattering of the randomly moving electrons and related transport characteristics.
\end{abstract}

Keywords: effective density of randomly moving (RM) electrons; diffusion coefficient; drift mobility; density-of-states (DOS); scattering; electronic defects; conductivity of metals

\section{Introduction}

The resistivity of crystalline elemental metals above the Debye's temperature increases linearly with temperature, and this behavior is usually explained by charge carriers scattering due to lattice atom vibrations: due to an increase of the magnitude of the electron-phonon scattering cross-section [1-9]. It has also been proposed that all valence electrons in the conduction band can freely move and can be scattered, but this proposition is completely wrong and contradicts the Fermi-Dirac statistics.

It is well known that the Pauli exclusion principle and the Fermi-Dirac statistics for electrons explain the experimental results of the electronic heat capacity of metals: why metals and isolators have around the same heat capacity. The resolution of this paradox is one of the greatest success of the Sommerfeld's model [1,10,11]. The main conclusion from this model is that only a small part of the conduction band electrons can move randomly, that their energy is close to the energy of Fermi level, and that electrons which energy is well below the Fermi level energy cannot change their energy because all neighbor energy levels are occupied. On the other hand, the main uncertainty of the Sommerfeld's model is that it does not strictly define the effective density of the randomly moving (RM) electrons, which can be scattered and produce the Brownian motion, and which can be affected by external fields. Moreover, by using this model with the spherical Fermi surface, it was pointed out that this model follows the Drude conductivity model of metals, but for metals the latter cannot be applicable in principle. There also are uncertainties in the determination of both the density-of-states (DOS) at the Fermi level energy and the Fermi level energy because the Fermi surfaces for many metals are not spherical and they have very composite forms [12].

There are many questions, for example, how to get the total density of the electrons in the conduction band having the Hall effect data of metals and considering that only small part of electrons in the conduction band takes part in the conduction; how to strictly define the density of the RM electrons and their related kinetic characteristics; and are the valence electrons in metals really free? 
This critical summary is mainly addressed in the application of the effective density of the RM electrons for the description of the basic electron transport characteristics in crystalline elemental metals.

\section{Results and Discussion}

\subsection{The Effective Density of RM Electrons in Crystalline Metals and Related Characteristics}

The total density $n$ of the electrons in the conduction band is described by DOS $g(E)$ in the unit volume as:

$$
n=\int_{0}^{\infty} g(E) f(E) \mathrm{d} E,
$$

where the Fermi distribution function is:

$$
f(E)=1 /[1+\exp (E-\eta) / k T] \approx 1 /\left[1+\exp \left(E-E_{\mathrm{F}}\right) / k T\right],
$$

here $\eta$ is the chemical potential, and $\eta \approx E_{\mathrm{F}}$ (here $E_{\mathrm{F}}$ is the Fermi level energy) because the difference between these quantities is only about $0.01 \%$ at room temperature; $k$ is the Boltzmann's constant; and $T$ is the absolute temperature.

The density of the free randomly moving (RM) electrons depends not only on the DOS $g(E)$ of the electrons in the conduction band and Fermi distribution function $f(E)$, but it also depends on the probability $f_{1}(E)=1-f(E)$ that at a given temperature $T$ the electron can be thermally scattered or change its energy under the influence of the external field.

Therefore, the effective density of the RM electrons $n_{\text {eff }}$ is defined as [13,14]:

$$
n_{\mathrm{eff}}=\int_{0}^{\infty} g(E) f(E)[1-f(E)] \mathrm{d} E=k T \int_{0}^{\infty} g(E)(-\partial f(E) / \partial \eta) \mathrm{d} E .
$$

This Equation is valid for homogeneous materials both with degenerate and nondegenerate electron gas. From this Equation, it also follows that the term:

$$
p(E)=(-\partial f(E) / \partial E)=f(E)[1-f(E)] / k T
$$

is the probability density function of energy $E$ of free RM electrons. The function $p(E)$ meets all requirements of the probability theory and is in agreement with the Pauli exclusion principle. The probability density function $p(E)$ is presented in Figure 1 . The area restricted by the dashed line is equal to 1 , and the effective width of the $p(E)$ in energy is $\Delta E_{\text {eff }}=4 k T$. Thus, the effective density of RM electrons is stochastic quantity, while the total density of valence electrons in the conduction band is a constant quantity.

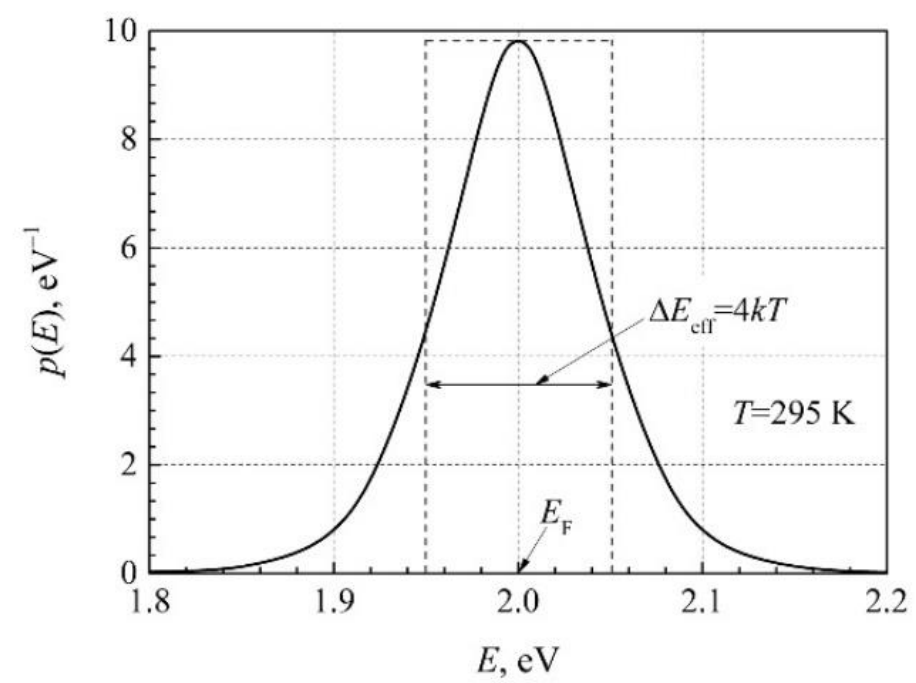

Figure 1. Illustration of the probability density function $p(E)$ dependence on the energy of the RM electrons near the Fermi level energy $E_{\mathrm{F}}=2 \mathrm{eV}$ at $T=295 \mathrm{~K}$. 
The average value of any random function $x(E)$, depending on the energy $E$ of the RM electrons, can be completely determined as:

$$
<x(E)>=\int_{0}^{\infty} x(E) p(E) g(E) d E .
$$

For materials (semiconductors) with non-degenerate electron gas, the probability $[1-f(E)] \approx 1$ because $f(E)<<1$, and all electrons in the conduction band take part in random motion, and their density is described as (1). It is the case when the classical statistics are valid. In the case of highly degenerate electron gas, the relation (3) can be presented in the following form:

$$
n_{\text {eff }}=g\left(E_{F}\right) k T<<n,
$$

where $g\left(E_{F}\right)=g(E)$ at $E=E_{F}$. There it can be pointed out that $n_{\text {eff }}$ at a given temperature $T$ is only determined by the DOS value at the Fermi surface, and it does not depend on the DOS distribution form in the conduction band, and on the form of Fermi surface, i.e., this relation is also valid for elemental transition metals. The $g\left(E_{F}\right)$ values can be obtained from the experimental data of the electronic heat capacity measurements $[15,16]$ :

$$
C_{\mathrm{el}}=\left(\pi^{2} / 3\right) g\left(E_{F}\right) k^{2} T=\gamma T \text {. }
$$

The electronic heat capacity parameter $\gamma$ for all elemental metals is determined and tabulated $[15,16]$. If we use the Sommerfeld's model based on the spherical Fermi surface we will get the DOS values $g\left(E_{F}\right)$ with very large uncertainty for metals with composite Fermi surfaces $[13,14]$. The relation between the density of the RM electrons and the total density of the valence electrons is shown in Figure 2a.
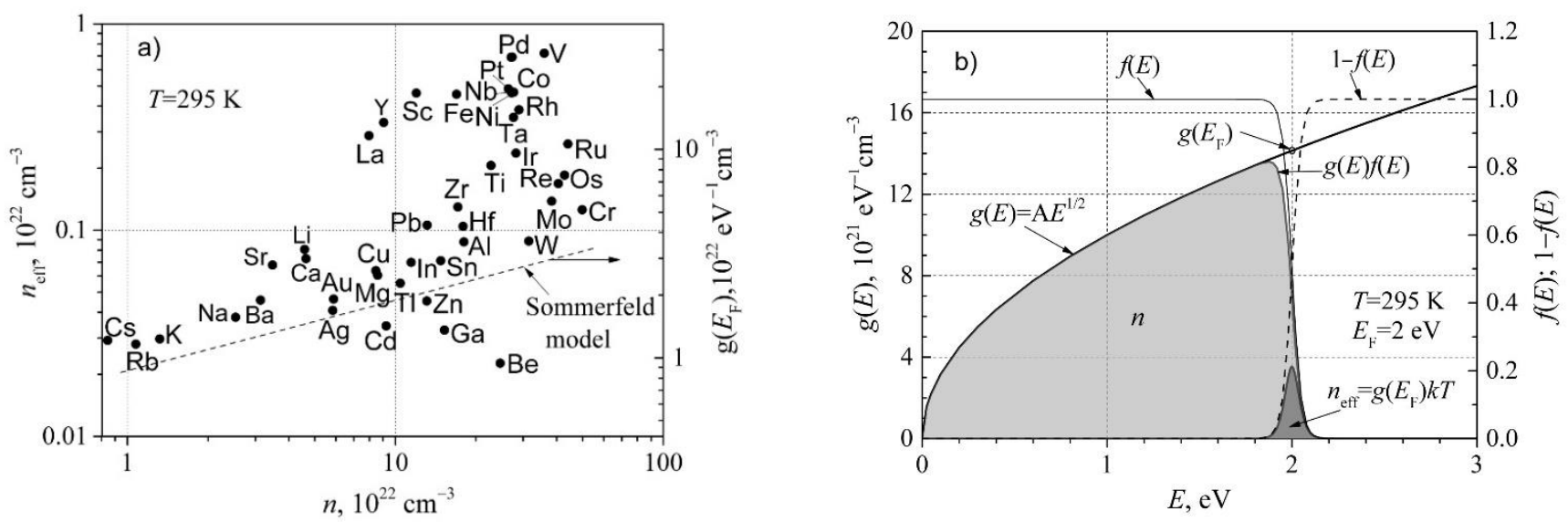

Figure 2. (a) The DOS at the Fermi level energy $g\left(E_{F}\right)$ (right scale) and the effective density of the RM electrons $n_{\text {eff }}($ left scale at $T=295 \mathrm{~K}$, calculated by Equation (3)) distribution on the total density of the valence electrons in the conduction band for elemental metals. The dash line (right scale) is under Sommerfeld's model. The data $g\left(E_{F}\right)$ are calculated by Equation (7) by using the electronic heat capacity values $[15,16]$. (b) Illustration of the DOS $g(E)$ and $g(E) f(E)$ functions (left scale) on the energy of the electrons in the conduction band with parabolical energy band. The light grey area represents the total density $n$ of the valence electrons (calculated by Equation (1)), and the dark grey area represents the effective density $n_{\text {eff }}$ of the RM electrons (calculated by Equation (3)). There are also presented the Fermi functions $f(E)$ and $[1-f(E)]($ right scale). Figure 2a with permission of the Nova Science Publishers.

In agreement with quantum mechanics, the electrons in the ideal periodicity crystal are arranged in energy bands according to the Bloch waves. Each primitive cell exactly contributes only one independent value of the wave vector to each energy band $[2,15,17]$. In the three-dimensional case, there are $2 \mathrm{~N}$ independent orbitals in each energy band, where $N$ is the number of primitive cells in the sample with the volume $V$. It can be here pointed out that the obtained result does not depend on both atom or electron masses. If an 
electron energy belongs to the conduction band, it does not automatically (by itself) mean that it can randomly move or can be scattered by thermal lattice vibrations. A schematic illustration of Equations (1)-(4) and DOS $g(E)$ functions for the parabolic energy band (for metals with spherical Fermi surface) is shown in Figure 2b.

The dark gray area represents the effective density of RM electrons $n_{\text {eff }}$ (estimated by Equation (3)); these RM electrons can be scattered and produce the Brownian motion and change their energy, but the other part of the electrons $\left(n-n_{\text {eff }}\right)$ cannot be scattered and change their energy. The probability density function that these electrons can be scattered is equal to zero. Moreover, these electrons are not affected by lattice thermal vibrations, because the variance of the thermal energy for these electrons is $\left\langle\Delta E^{2}\right\rangle=0$. Therefore, that part of the electrons is localized and bonded to their native ions. The solid line in Figure 2a has been calculated by using well-known Sommerfeld formula $g\left(E_{\mathrm{F}}\right)=\left[m /\left(\hbar^{3} \pi^{2}\right)\right] \sqrt{2 m E_{\mathrm{F}}}$ with $m$ equal to the free electron mass. To get the agreement of Sommerfeld formula with experimental data, it is used a particular effective mass of the DOS of elemental metals $[1,8,11]$, but the DOS in the energy band is caused only by the number of primitive cells in the sample $[2,15,17]$.

The average energy of the valence electron in the metal is smaller than in the isolated atom. This decrease in the energy of the valence electrons in metals causes the binding energy of atoms in crystalline elemental metals $[1,18]$. The valence electron wave functions overlap substantially with that of the neighbor atoms $[2,11,15]$, but they remain associated with the native atoms: The valence electron charge cloud density is concentrated near the parent ions. Only the valence electrons with energies that are close to the Fermi level energy due to lattice ion vibrations can be released, produce the Brownian motion in the metal crystal, and can leave the native atoms; they become free and can randomly move in the crystalline metal. Thus, the free electron term can be used only for free RM electrons, the other part $\left(n-n_{\text {eff }}\right)$ of the valence electrons is localized near the native ions. Here we want to point out that part of the electrons $\left(n-n_{\text {eff }}\right)$ is not free yet for alkali metals though their Fermi surfaces are almost spherical.

\subsection{Diffusion Coefficient and Drift Mobility of RM Electrons}

The electrical conductivity $\sigma$ and the free electron diffusion coefficient $D$ are related by the following general expression $[19,20]$ :

$$
\sigma=q^{2} D(\partial n / \partial \eta)_{T}
$$

After a simple calculation of the derivative $n$ on the chemical potential $\eta$, the following expression is obtained:

$$
\sigma=\frac{q^{2} D}{k T} \int_{0}^{\infty} g(E) f(E)[1-f(E)] \mathrm{d} E=\frac{q^{2} D}{k T} n_{\text {eff }}
$$

where $n_{\text {eff }}$ is described by (3). Equation (9) shows that the conductivity in all cases is determined by the effective density of RM electrons. The same expression is also obtained from the Boltzmann kinetic Equation $[4,21]$ and from the description of the electric thermal noise [13].

On the other hand, the conductivity can be described as:

$$
\sigma=q \mu_{\mathrm{drift}} n_{\mathrm{eff}}
$$

where $\mu_{\text {drift }}$ is the drift mobility of the RM electrons. From relations (9) and (10) it follows the Einstein's relation between the diffusion coefficient and the drift mobility of the free RM electrons:

$$
D / \mu_{\mathrm{drift}}=k T / q .
$$

Equations (9)-(11) are valid for all homogeneous materials with a single type of charge carrier at any degeneracy degree. The relationships between the conductivity, 
diffusion coefficient, and drift mobility of the RM electrons for elemental metals at $295 \mathrm{~K}$ are presented in Figure 3a. From this figure it follows that the drift mobility for most of the elemental metals is many times larger than the Hall mobility [22,23].
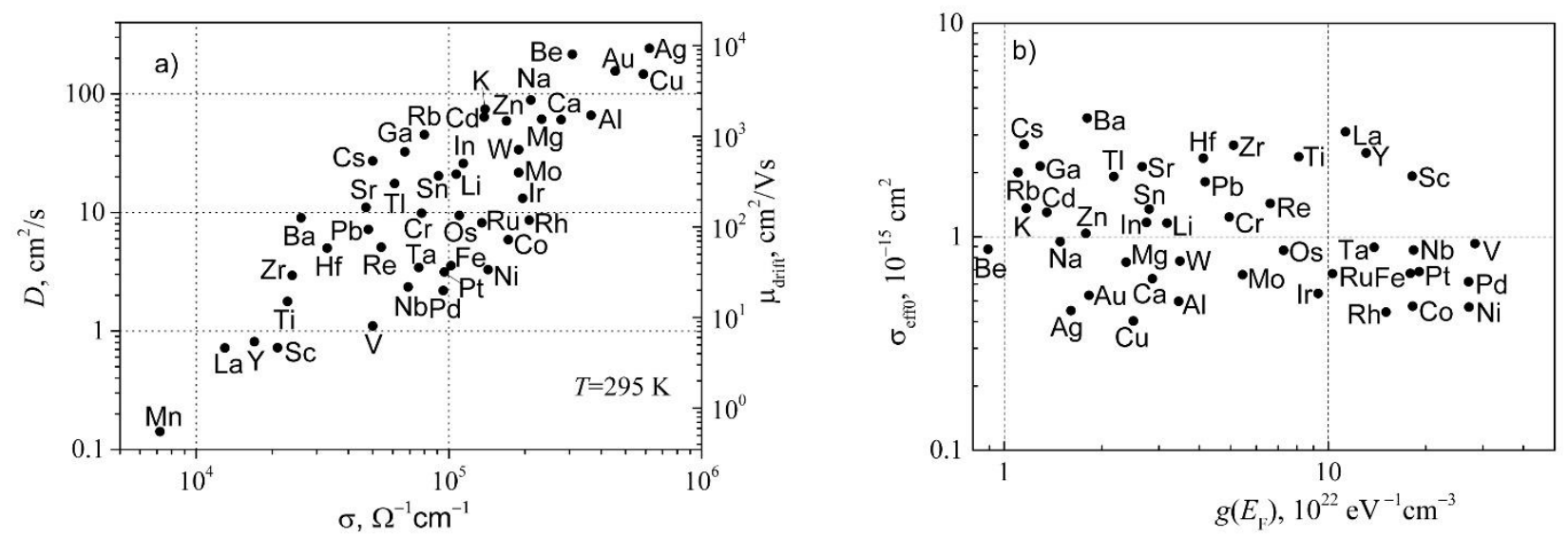

Figure 3. (a) Relationship between conductivity, diffusion coefficient (left scale, calculated by Equation (12) by using the experimental conductivity data) and drift mobility (right scale, calculated by Equation (10)) of the RM electrons for elemental metals at $T=295 \mathrm{~K}$ (the conductivity values are taken from $[15,16]$ ). (b) The effective scattering cross-section $\sigma_{\text {effo }}$ caused by electronic defects of elemental metals distribution on DOS at Fermi surface at $T=295 \mathrm{~K}$. Figure 3 with permission of the Nova Science Publishers.

For elemental metals, the conductivity (9) can be described as

$$
\sigma=q^{2} g\left(E_{\mathrm{F}}\right) D=(1 / 3) q^{2} g\left(E_{\mathrm{F}}\right) v_{\mathrm{F}}^{2} \tau_{\mathrm{F}},
$$

where $v_{\mathrm{F}}$ and $\tau_{\mathrm{F}}$ are, respectively, the velocity and relaxation time of the RM electrons at the Fermi surface.

\subsection{The Scattering of the RM Electrons and Their Mean Free Path}

From Equation (12) it follows that the diffusion coefficient is related to at least five transport parameters of the RM electrons:

$$
D=\frac{\sigma}{q^{2} g\left(E_{F}\right)}=\frac{1}{3} v_{\mathrm{F}}^{2} \tau_{\mathrm{F}}=\frac{1}{3} l_{\mathrm{F}} \tau_{\mathrm{F}},
$$

where $l_{\mathrm{F}}$ is the mean free path of the RM electrons.

The most important parameter characterizing the scattering mechanism of the RM electrons is their mean free path. According to the quantum mechanics, the free electrons can freely move in the ideal periodic lattice of the metal crystal without any scattering by ions $[1,5,15,17,21]$. Therefore, the scattering of free electrons can be only in spots where there are distortions of the periodicity of the potential energy of the ideal lattice structure. The resistivity of metals is caused due to the scattering of free electrons by impurities, vacancies, interstitial atoms, dislocations, boundaries of grains, and the surface. The electron mean free path due to the named defects almost does not depend on temperature, and their dominance appears at low temperatures. In the temperature range above the Debye's temperature, the mean free path changes as $1 / T$, while below the Debye's temperature it usually changes as $T^{-5}$. Considering that the effective density of the free RM electrons increases with temperature [ $n_{\text {eff }}=g\left(E_{\mathrm{F}}\right) k T$ ], another explanation mechanism is needed to explain the electron scattering cross-section by lattice vibrations.

Now, let us return to the Fermi distribution function. The average effective density of the RM electrons in elemental metals is $n_{\text {eff }}=g\left(E_{\mathrm{F}}\right) k T$, which are produced by thermal vibrations of some of the atoms, i.e., by atoms which have valence electrons with energy 
near to Fermi level energy. It means that on average there is an effective density of atoms $N_{\text {eff }}$, which can generate free RM electrons, and it may be expressed as

$$
N_{\text {eff }}=n_{\text {eff }}=g\left(E_{\mathrm{F}}\right) k T \text {. }
$$

These $N_{\text {eff }}$ atoms generate free RM electrons, which lose the parent atoms to large distances of order of the electron mean free path and also produce both not completely screened positive ions and local distortions in the periodical distribution of the charge density. Considering that these defects do not produce any distortion in the lattice structure, such defects are named as electronic defects [22,23].

The other part $\left(N_{\mathrm{at}}-N_{\text {eff }}\right)$ of atoms that have valence electrons with energy well below the Fermi level energy does not have sufficient thermal vibration energy to excite RM electrons near to Fermi level energy (here $N_{\mathrm{at}}$ is the density of metal atoms). In other words, the part $\left(n-n_{\mathrm{eff}}\right)$ of the valence electrons which energy is lower than $E_{F}$ by at least several units of $k T$, due to Pauli exclusion principle and Fermi-Dirac statistics, is not affected by lattice vibrations, because all neighbor energy levels are occupied. It is the most important property of such electrons. Moreover, this part of electrons has no influence on the motion of the free RM electrons. Thus, the Pauli exclusion principle drastically reduces the number of processes by which valence electrons can interact and be scattered by ions and other electrons. The vibrations of the $\left(N_{\mathrm{at}}-N_{\text {eff }}\right)$ atoms can be interpreted as neutral atom vibrations: the part $\left(n-n_{\mathrm{eff}}\right)$ of valence electrons is sufficiently tightly bonded to their parent ions, and they move together with ions because they cannot change their energy. Usually, the movement of electrons without the energy changing corresponds to their stationary motion.

Therefore, with a temperature increase, the thermal vibrations of the lattice ions stimulate the increase of the RM electron density $n_{\mathrm{eff}}$, and at the same time produce the same density $N_{\text {eff }}$ of the local distortion spots (electronic defects) of the potential (or charge density) periodicity.

Then the electron mean free path $l_{\mathrm{F}}$ of the RM electrons in metals can be described as:

$$
l_{\mathrm{F}}=v_{\mathrm{F}} \tau_{\mathrm{F}}=1 /\left(\sigma_{\text {eff }} N_{\text {eff }}\right)=1 /\left[\sigma_{\text {eff }} g\left(E_{F}\right) k T\right],
$$

where $v_{\mathrm{F}}$ and $\tau_{\mathrm{F}}$ are, respectively, the electron velocity and their relaxation time at the Fermi surface; and $\sigma_{\text {eff }}$ is the effective scattering cross-section of the RM electrons by electronic defects. On the other hand, the average relaxation time can be described as:

$$
\tau_{\mathrm{F}}=1 /\left(\sigma_{\mathrm{eff}} N_{\text {eff }} v_{\mathrm{F}}\right)=1 /\left[\sigma_{\mathrm{eff}} g\left(E_{F}\right) v_{\mathrm{F}} k T\right] .
$$

From Equations (15) and (16) it follows that the effective scattering cross-section of the RM electrons at temperatures over Debye's temperature does not depend on temperature.

As it is shown in [23], the effective scattering cross-section of electrons $\sigma_{\text {eff }}$ by electronic defects depends on the ratio of the exchange of the thermal energies between the electronic defect and RM electron:

$$
\sigma_{\text {eff }}=\sigma_{\text {effo }} \eta_{\mathrm{ph}}(T / \Theta),
$$

where $\sigma_{\text {effo }}$ can be evaluated from (15) at room temperature; $\Theta$ is the Debye's temperature; and $\eta_{\mathrm{ph}}(T / \Theta)$ is the phonon mediation factor accounting for the RM electrons scattering by electronic defects [23]:

$$
\eta_{\mathrm{ph}}(T / \Theta)=(T / \Theta)^{4} \int_{0}^{\Theta / T} \frac{4 x^{5} \mathrm{~d} x}{\left(e^{x}-1\right)\left(1-e^{-x}\right)} .
$$

The effective scattering cross-section of electrons $\sigma_{\text {eff }}=\sigma_{\text {eff } 0}$ distribution on the DOS at the Fermi surface at $T_{0}=295 \mathrm{~K}$ evaluated by (15), and by using the electron mean free 
path data from [23] for elemental metals, is shown in Figure 3b. It is seen that the obtained cross-section values $\sigma_{\text {effo }}$ on average correspond to atom cross-section values.

The resultant average relaxation time $\tau_{\text {res }}$ in a wide temperature range can be described as:

$$
1 / \tau_{\text {res }}=\left(1 / \tau_{\text {eff }}\right)+\left(1 / \tau_{\text {imp }}\right),
$$

where $\tau_{\text {eff }}=1 /\left[\sigma_{\text {eff } 0} \eta_{\mathrm{ph}}(T / \Theta) g\left(E_{\mathrm{F}}\right) v_{\mathrm{F}} k T\right]$, and $\tau_{\text {imp }}=1 /\left(\sigma_{\mathrm{imp}} N_{\mathrm{imp}} v_{\mathrm{F}}\right)$ is the electron relaxation time due to their scattering at low temperature by various impurities and structure defects of the lattice; $\sigma_{\mathrm{imp}}$ is the electron scattering cross-section by impurities; and $N_{\text {imp }}$ is the impurity density.

Then the resistivity of the elemental metal in the overall temperature range can be described as:

$$
\rho(T)=\rho_{0}+\rho\left(T_{0}\right) \cdot\left(T / T_{0}\right) \cdot \eta_{\mathrm{ph}}(T / \Theta),
$$

where $\rho_{0}$ is the residual resistivity due to electron scattering by imperfections of the lattice defects, and $\rho\left(T_{0}\right)$ is the resistivity at $T=295 \mathrm{~K}$.

An illustration of the applicability of the relation (20) for the estimation of the resistivity is presented in Figure $4 \mathrm{a}$ for $\mathrm{Sr}, \mathrm{Au}, \mathrm{Cu}, \mathrm{Mo}$, and $\mathrm{Al}$, and in Figure $4 \mathrm{~b}$ for $\mathrm{Pd}, \mathrm{V}, \mathrm{Ag}, \mathrm{Zn}$, and $\mathrm{W}$ in temperature range from 1 to $1000 \mathrm{~K}$.
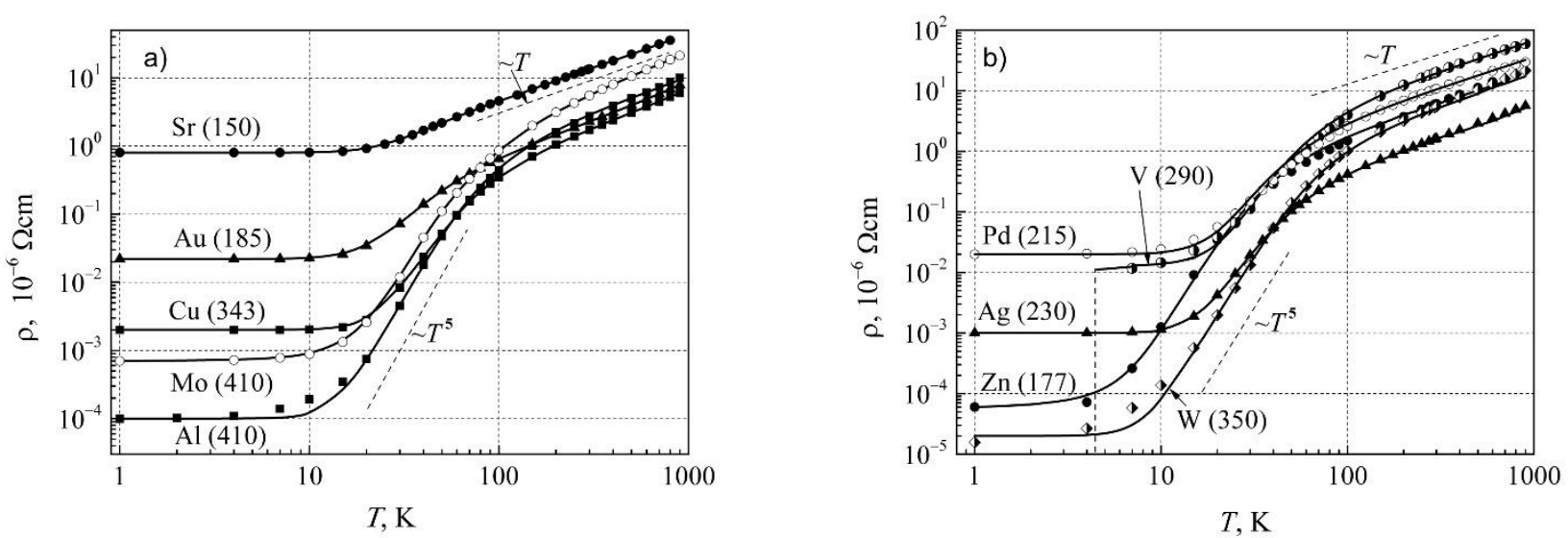

Figure 4. Electrical resistivity of $\mathrm{Sr}, \mathrm{Au}, \mathrm{Cu}, \mathrm{Mo}$, and $\mathrm{Al}(\mathbf{a})$; and $\mathrm{Pd}, \mathrm{V}, \mathrm{Ag}, \mathrm{Zn}$, and $\mathrm{W}(\mathbf{b})$ dependence on temperature (dots are experimental data [16], the solid lines are calculation results by Equation (20); in brackets near the metal chemical sign there is the Debye's temperature $\Theta$ in K used for calculation).

\section{Conclusions}

It is shown that the electron transport characteristics in elemental metals are caused by randomly moving electrons at the Fermi surface, but not by the total density of the electrons in the conduction band. The creation of the RM electrons by the lattice vibrations produces the same number of electronic defects, which causes the scattering of the RM electrons. It allows to estimate the real characteristics of RM electrons of crystalline elemental metals, such as their diffusion coefficient, drift mobility, average relaxation time, and average scattering cross-section caused by electronic defects. It is shown that the Einstein relation between the diffusion coefficient and drift mobility of the free RM electrons is valid at any degree of degeneracy of electron gas. It is also shown that at temperatures over the Debye's temperature, the scattering cross-section of the RM electrons does not depend on temperature. The valence electrons, which energy is lower by at least several units of $k T$ than the Fermi level energy, can only move around the native ions because the energy of ion thermal vibration is not sufficient to excite them into the range of the Fermi level energy with free energy states. 
Author Contributions: V.P. is an author of theoretical concept and derivations, and E.Ž. performed calculations and visualization of data. The manuscript is written through contributions of both authors. Both authors have given approval to the final version of the manuscript.

Funding: This research received no external funding.

Data Availability Statement: Data are provided in the figures of the article.

Conflicts of Interest: The authors declare no conflict of interest.

\section{References}

1. Ashcroft, N.W.; Mermin, N.D. The Sommerfeld Theory of Metals. In Solid State Physics; Harcourt College Publ.: New York, NY, USA, 1976

2. Myers, H.P. Introductory Solid State Physics; Taylor \& Francis Publishers: London, UK, 2009.

3. Ziman, J.M. Electrons and Phonons. In The Theory of Transport Phenomena in Solids; Oxford University Press: Oxford, UK, 2001.

4. Abrikosov, A.A. Fundamentals of the Theory of Metals; North-Holland Publication: Amsterdam, The Netherlands, 1988.

5. Kaveh, M.; Wiser, N. Electron-electron scattering in conducting materials. Adv. Phys. 1984, 33, 257-372. [CrossRef]

6. Lundstrom, M. Fundamentals of Carrier Transport; Cambridge University Press (Online): Cambridge, UK, 2014. [CrossRef]

7. Rossiter, P.L. The Electrical Resistivity of Metals and Alloys; Cambridge University Press (Online): Cambridge, UK, 2014.

8. Schulze, G.E.R. Metallphysik; Akademie-Verlag: Berlin, Germany, 1967.

9. Wilson, A.H. The Theory of Metals; Cambridge University Press: Cambridge, UK, 1958.

10. Sommerfeld, A.; Bethe, H. Elektronentheorie der Metalle; Springer: Berlin, Germany, 1967.

11. Mizutani, U. Introduction to the Electron Theory of Metals; Cambridge University Press (Online): Cambridge, UK, 2014.

12. Cracknell, A.P.; Wong, K.C. The Fermi Surfaces: Its Concept, Determination, and Use in the Physics of Metals; Clarendon Press: Oxford, UK, 1973.

13. Palenskis, V. Drift mobility, diffusion coefficient of randomly moving charge carriers in metals and other materials with degenerate electron gas. World J. Cond. Matt. Phys. 2013, 3, 73-81. [CrossRef]

14. Palenskis, V. The effective density of randomly moving electrons and related characteristics of materials with degenerate electron gas. AIP Adv. 2014, 4, 047119. [CrossRef]

15. Kittel, C. Introduction to Solid State Physics; John Wiley and Sons, Inc.: New York, NY, USA, 1976.

16. Elements Handbook. Available online: http://www.knowledgedoor.com/ (accessed on 1 April 2021).

17. Blokhintsev, D.L. Quantum Mechanics; D. Reidel Publishing Company: Dordrecht, The Netherlands, 1964.

18. Blakemore, J.S. Solid State Physics; Cambridge University Press: Cambridge, UK, 1985.

19. Bonch-Brujevitch, V.L.; Kalashnikov, S.G. The Physics of Semiconductors; Nauka Press: Moscow, Russia, 1990. (In Russian)

20. Bisquert, J. Interpretation of electron diffusion coefficient in organic and inorganic semiconductors with broad distributions of states. Phys. Chem. Chem. Phys. 2008, 10, 3175. [CrossRef] [PubMed]

21. Dugdale, J.S. The Electrical Properties of Disordered Metals; Cambridge University Press: Cambridge, UK, 1985. [CrossRef]

22. Wert, C.; Thomson, R.M. Physics of Solids; McGraw-Hill Book Company: New York, NY, USA, 1964.

23. Palenskis, V.; Žitkevičius, E. Analysis of transport properties of randomly moving electrons in metals. Mater. Sci. 2020, 26, 147-153. [CrossRef] 